\title{
REVIEW ARTICLE OPEN Spaceflight-induced neuroplasticity in humans as measured by MRI: what do we know so far?
}

\author{
Angelique Van Ombergen ${ }^{1,2,3}$, Steven Laureys ${ }^{4}$, Stefan Sunaert ${ }^{5}$, Elena Tomilovskaya ${ }^{6}$, Paul M. Parizel ${ }^{7}$ and Floris L. Wuyts ${ }^{1,3}$
}

Space travel poses an enormous challenge on the human body; microgravity, ionizing radiation, absence of circadian rhythm, confinement and isolation are just some of the features associated with it. Obviously, all of the latter can have an impact on human physiology and even induce detrimental changes. Some organ systems have been studied thoroughly under space conditions, however, not much is known on the functional and morphological effects of spaceflight on the human central nervous system. Previous studies have already shown that central nervous system changes occur during and after spaceflight in the form of neurovestibular problems, alterations in cognitive function and sensory perception, cephalic fluid shifts and psychological disturbances. However, little is known about the underlying neural substrates. In this review, we discuss the current limited knowledge on neuroplastic changes in the human central nervous system associated with spaceflight (actual or simulated) as measured by magnetic resonance imaging-based techniques. Furthermore, we discuss these findings as well as their future perspectives, since this can encourage future research into this delicate and intriguing aspect of spaceflight. Currently, the literature suffers from heterogeneous experimental set-ups and therefore, the lack of comparability of findings among studies. However, the cerebellum, cortical sensorimotor and somatosensory areas and vestibular-related pathways seem to be involved across different studies, suggesting that these brain regions are most affected by (simulated) spaceflight. Extending this knowledge is crucial, especially with the eye on long-duration interplanetary missions (e.g. Mars) and space tourism.

npj Microgravity (2017)3:2 ; doi:10.1038/s41526-016-0010-8

\section{INTRODUCTION}

More than 50 years of manned spaceflight have taught us that space is a hostile environment for human health; microgravity, ionizing radiation, absence of circadian rhythm, confinement and isolation are just some of the stressors space travelers encounter. ${ }^{1,2}$ Obviously, all of the latter can have an impact on human physiology and lead to detrimental changes. ${ }^{3}$ An example of this is the microgravity-induced cephalic fluid shift, which has been thought to cause to a wide range of symptoms such as increased intracranial pressure, visual impairment (named the visual impairment intracranial pressure syndrome, VIIP syndrome) $)^{4,5}$ and alterations in cerebral oxygenation ${ }^{6}$ and cerebral blood flow $(\mathrm{CBF})^{7,8}$ (for a full synthesis on spaceflight-induced cephalic fluid shift, readers are referred to. ${ }^{9}$

It is important to acquire insight into the precise effect of spaceflight as this can aid in the development of adequate countermeasures and guarantee safety and efficiency in future space missions. Some organ systems have been studied thoroughly under space conditions, such as the cardiovascular, ${ }^{10}$ immune ${ }^{11,12}$ and musculoskeletal systems. ${ }^{13,14}$ Although there is an increasing interest on the effect of spaceflight on the human central nervous system (CNS), ${ }^{15,16}$ up to date, not much is known about the functional and morphological effects of microgravity on the human CNS. Previous studies have already shown that CNS changes occur during and after spaceflight in the form of neurovestibular problems, ${ }^{17,18}$ alterations in cognitive function and sensory perception, ${ }^{19}$ problems with motor function, ${ }^{20}$ cephalic fluid shift ${ }^{9}$ and psychological disturbances. ${ }^{21}$ For example, neurovestibular problems originate partially at the level of the peripheral vestibular organ that suddenly is deprived of the sense of gravity, ${ }^{22-24}$ so an intravestibular conflict emerges between the different angular and linear acceleration detectors. Therefore, one could hypothesize that this may also have an effect on the vestibular nuclei in the brain as well as on the cortical projections where sensory integration takes place between 'disturbing' vision, 'altered' proprioception and 'conflicting' vestibular information, such as the insular cortex, the temporoparietal junction and the thalamus. ${ }^{25,26}$ In addition, it is known that the primary somatosensory and the somatosensory association cortical networks are involved in proprioception. ${ }^{27}$ Zerogravity induced modifications in these network interactions could therefore underlie the deficits in sensory perception as seen in astronauts and vice versa. ${ }^{28}$ Also, the cerebellum is known to be involved in fine motor control, coordination and equilibrium ${ }^{29}$ and changes in cerebellar function and connectivity could therefore explain typically-seen motor coordination and movement-timing problems during and after spaceflight. ${ }^{28}$

In general, literature on the impact of spaceflight on space travelers has mainly focused on the extra-cerebral or peripheral systems, e.g. the musculoskeletal and the cardiovascular system.

\footnotetext{
${ }^{1}$ Antwerp University Research Centre for Equilibrium and Aerospace (AUREA), University of Antwerp, Groenenborgerlaan 171 , Antwerp 2020, Belgium; ${ }^{2}$ Faculty of Medicine and Health Sciences, University of Antwerp, Universiteitsplein 1, Wilrijk (Antwerp) 2610, Belgium; ${ }^{3}$ Faculty of Sciences, Department of Biomedical Physics, University of Antwerp,

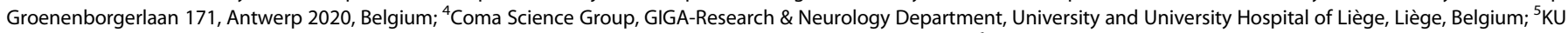

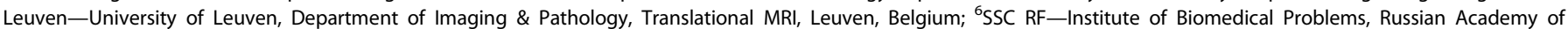
Sciences, Moscow, Russia and ${ }^{7}$ Department of Radiology, Antwerp University Hospital \& University of Antwerp, Antwerp, Belgium Correspondence: Floris L Wuyts (floris.wuyts@uantwerpen.be)
} 
Yet, studies on CNS dysfunction are scarce. However, in the past few years more and more interest has been attributed to this topic. The latter is probably due to recent advances in structural and functional neuroimaging techniques over the past 20 years leading to a growing role of these technologies in Earth-bound medicine. Additionally, the increasing interest in interplanetary missions adds to the importance to probe the changes occurring in the human brain in relation to short- and long-duration spaceflight.

\section{Aim of this review}

Previous reviews on spaceflight-induced neuroplasticity, ${ }^{30-34}$ dating from the 1990 's or early 2000 , are largely based on animal studies and do not include more recent findings from more advanced neuroimaging techniques. Furthermore, the effect of space analogs -in particular head-down bed rest (HDBR)- on the human brain has received increasing interest in the past few years and has resulted in novel findings, some of which are translatable to long-duration spaceflight. An updated overview of this emerging literature could help to synthesize our current understanding, as well as to address the current shortcomings in order to direct and enhance future research.

\section{Neuroplasticity and how to measure it}

Neural plasticity or neuroplasticity can be defined as the capability of the brain to alter its structure or function in response to exposure to new stimuli or environments. It is a crucial underlying component of skill learning in healthy individuals (i.e. learningdependent or experience-dependent neuroplasticity) and functional recovery after injury. ${ }^{35}$ Neural plasticity can take place at several levels: from synaptic plasticity at the (sub)cellular level to plasticity at the system and network level. ${ }^{35}$ In this review, we will focus on systems plasticity across neural networks in human beings. Brain plasticity of the CNS can be studied with a number of methods. Examples of techniques commonly used in neuroplasticity studies are electroencephalography (EEG)/evoked potentials (ERPs), structural and functional magnetic resonance imaging (MRI) and transcranial magnetic stimulation (TMS). These techniques can be used to study the cortical dynamics, e.g. magnitude of task-related or resting-state neural activity, changes in activity patterns, representational map size and cortical excitability. Other commonly used techniques include positron emission tomography (PET) and magnetic resonance spectroscopy, but up until now, no space-related studies have been carried out with the latter techniques, so we will only describe the applied techniques related to real and simulated spaceflight.

When it comes to spaceflight, EEG is the most commonly used technique. This is associated with the portability of EEG and the fact that this technique can easily be used in extreme environments. ${ }^{36}$ In EEG, electrical activity of the brain is monitored and measured by placing multiple electrodes along the scalp. Examples of the use of EEG in regards to spaceflight are the studies on electrocortical activity in astronauts during spaceflight $^{37}$ or in subjects during parabolic flights. ${ }^{38}$ EEG has a high temporal resolution, but on the contrary, it has a low spatial resolution making it tricky to attribute EEG findings to a precise cortical or subcortical region. Current state of the art neuroimaging techniques such as MRI, as further described below, have a high spatial sensitivity and therefore allow a detailed assessment of brain structure and function. ${ }^{39}$ We will not go into detail on EEG-based space studies on neuroplasticity, but we will focus on spaceflight-induced neuroplasticity as measured by MRI. However, EEG studies have been proven to be very useful in better understanding the effect of spaceflight and microgravity on the human brain and ideally, would be combined with functional MRI in a multimodal fashion to cover both temporal and spatial aspects of neuroplasticity as good as possible. Readers are referred to Marušič et al. for a recent and thorough review on EEG-based neuroplasticity studies in relation to spaceflight, microgravity and hypergravity. ${ }^{36}$

$\mathrm{MRI}$ is an imaging technique that allows measuring structural, functional, metabolic and vascular events in vivo. An example of an anatomical MRI-technique is volumetric T1-weighted anatomical imaging to assess regional differences of a specific brain region, i.e. gray matter (GM), WM and cerebrospinal fluid (CSF), between groups. A common technique to perform this type of brain morphometry is called 'voxel-based morphometry'. ${ }^{40}$ Another MRI technique is diffusion tensor imaging (DTI). DTI is based on the molecular Brownian motion (i.e. diffusion) of the water molecules in the brain. ${ }^{41,42}$ Several local microstructures such as myelin, cell membranes and other organelles will limit free diffusion in the brain. The DTI MRI technique uses this limitation of free diffusion by measuring the diffusion path of water molecules. In DTI, it is assumed that the signal in each voxel can be described as a diffusion tensor. This diffusion tensor will determine the orientation of the longest axis of the ellipsoid, which will be ideally aligned with the orientation of the underlying white matter architecture. From the diffusion tensor, several parameters can be defined, such as fractional anisotropy (FA) and mean diffusivity. Therefore, DTI allows, up to some extent, to study the underlying white matter (WM) structure and microstructural features. ${ }^{41,42} \mathrm{~A}$ semi-automated procedure can now be implemented to connect neighboring voxels where the diffusion tensor points towards each other, and by doing so the underlying WM bundle can be reconstructed. This process is called diffusion tensor tractography. ${ }^{43,44}$ Another technique called tract-based spatial statistics, an automated and observer-independent approach, allows to assess FA in the major WM tracts on a voxel-wise basis across groups of subjects. $^{45}$

Functional MRI (fMRI) is also a MRI-based technique in which stimulus or activity-induced brain patterns can be investigated. fMRI is based upon the fact that neural activation is associated with a local vascular response, constituting the blood-oxygen-level dependent (BOLD) signal. The magnitude of the BOLD-signal resembles the hemodynamic response and can indirectly be linked to the magnitude of neural activation in specific brain areas. fMRI has been crucial in the determination of functional organization in the human brain. ${ }^{46,47} \mathrm{~A}$ derivative of $\mathrm{fMRI}$ is the resting-state $\mathrm{fMRI}$ technique (rsfMRI) in which neural activity at rest, without any stimulus or activity, is measured. For a complete summary on the use of MRI-based techniques in neuroplasticity studies, readers are referred to. ${ }^{48}$

Lastly, TMS is a technique that allows stimulation of an area of the cortex non-invasively through the scalp by means of brief pulses, administered by a stimulation coil using time-varying magnetic fields. ${ }^{49}$ By doing so, alterations in cortical excitability can be induced and measured. For example, when TMS is applied over the primary motor cortex (M1), TMS can depolarize the corticospinal tracts and evoke contralateral muscle contractions. ${ }^{49}$ For a review on the use of TMS in neuroplasticity studies, readers are referred to e.g. ${ }^{49}$ or. ${ }^{50}$ TMS has been used previously to investigate corticospinal excitability in relation to hypergravity and microgravity, however, this was a preliminary investigation and data from only 3 parabolic flyers were included. ${ }^{51}$

\section{Ground-based space alternatives for human studies}

Research on humans in space is complicated, expensive and subject to several logistic and payload restrictions. In addition, only few subjects can be investigated at the same time, leading to reduced study power and limited generalization. Therefore, space researchers have developed Earth-based models in which some aspects of spaceflight can be simulated in order to set-up investigations on a bigger scale and by which the difficulties of actual space research can be overcome. 
Immersion was the first ground-based model ever used for investigating the consequences of spending time in a reduced gravity environment. Dry immersion involves immersing the subject in thermo-neutral water while being covered in an elastic waterproof fabric in order to keep the subject dry and to overcome the unpleasant consequences of long-term direct water exposure. ${ }^{52}$ Immersion is an adequate spaceflight alternative, since it mimics several spaceflight features such as 'supportlessness' (i.e. lack of a supporting structure against the body), centralization of bodily fluids, confinement, immobilization and hypokinesia. ${ }^{52}$ Although dry immersion is a good model, it is not (yet) widely implemented and so far, it has not been used to quantify the neural changes associated with it. For a more general review on dry immersion and its implementation, readers are referred to. 52

HDBR is an acceptable, reliable and the most implemented alternative to simulate most of the changes occurring due to spaceflight, both of a physiological ${ }^{53,54}$ and a psychological kind. ${ }^{55}$ In principle, HDBR consist of a subject being in a bed that is inclined with the head down ( $-6^{\circ}$ in most cases). This can be done for short-term investigations (e.g. 72 hours $i^{56}$ ) or long-term studies (e.g. 90 days $\mathrm{in}^{57}$ ). The head down tilt induces an upward fluid shift, similar to the one seen in space. Spaceflight-induced cephalic fluid shift is thought to cause a wide range of symptoms such as increased intracranial pressure, visual impairment (together named the VIIP syndrome), alterations in cerebral oxygenation and changes in CBF. In addition, HDBR is also characterized by immobilization, inactivity and confinement. This leads to equivalent alterations as seen in spaceflight in calcium homeostasis, musculoskeletal deterioration (e.g. muscle loss and changes in bone architecture) and a psychological load, respectively. For a review on bed rest and its application in space research, readers are referred to the publication by Pavy-Le Traon and colleagues. ${ }^{54}$

A third "ground-based" alternative to spaceflight is parabolic flight (PF). During a PF, a specific flight trajectory wherein the acceleration of the aircraft cancels the acceleration due to gravity is carried out. By doing so, normo-, hyper- and microgravity phases are alternatingly experienced by the subjects on board of the PF aircraft. The hypergravity phase precedes and follows the microgravity phase and is characterized by 1.5 to $1.8 \mathrm{~g}$ and lasts around $30-35 \mathrm{~s}$. The microgravity phase on the contrary resembles $0 \mathrm{~g}$ during which approximately $0 \mathrm{~g}$ is experienced lasting around 20-25 s (Fig. 1). In addition, the flight profile can be modified to fly parabolas of Martian gravity $(0.38 \mathrm{~g})$ and lunar gravity $(0.16 \mathrm{~g})$. In between parabolas, the aircraft flies in normal $1 \mathrm{~g}$ conditions. In general, one PF consists of 31 parabolas and lasts around 3 to $3.5 \mathrm{~h}$. For more information on the underlying dynamics of a PF, readers are referred to the paper of. ${ }^{58}$ Important to note is that PF is the only Earth-based method that allows researchers to conduct life science studies in microgravity.

Another approach to mimic spaceflight-related features, is to investigate human deployment analogs, such as Antarctic overwintering, undersea missions, etc... Sensory deprivation, high stress loads, confinement, isolation and shifted circadian rhythm are all replicated to high fidelity and therefore, these missions form an acceptable spaceflight analog (except for space-related changes in gravity). Furthermore, space mission simulation studies in the form of isolation missions, e.g. the MARS500 study, can also be used as a spaceflight analog, in particular to investigate the effects of long-term isolation and confinement. An example hereof is the assessment of peripheral and central (assessed by means of EEG) stress markers in the MARS500 mission. ${ }^{59}$

\section{Search method}

For this review, the Medline (PubMed) and EMBASE databases were searched for papers using the term "spaceflight", "microgravity", "bed rest", "PF", "dry immersion" or "head-down tilt" and "brain", "neuroplasticity", "neuro", "MRI", "DTI" or "fMRI" without restriction of publication date. Reference lists from retrieved articles were also searched manually for relevant publications that were not included in the lists created through the Medline database. Non-English studies were excluded. The abstracts of the resulting articles were screened to select the relevant articles, i.e. articles describing new findings on spaceflight-induced neuroplasticity or commenting on previously reported results in the field. Only studies on human subjects were included. As stated above, EEG-based studies were excluded from this review.

\section{OVERVIEW AND CRITICAL APPRAISAL OF THE CURRENT LITERATURE}

A synthesis and critical appraisal of the MRI-based studies included can be found in Table 1. For clarification, a summary of brain regions found to be affected in (simulated) microgravity can be found in Fig. 2.

\section{Neuroplasticity and spaceflight}

So far, there has only been one study examining the neuroplastic effects after actual spaceflight by means of MRI. In this singlesubject case study, it was shown, by means of rsfMRl, that longduration spaceflight is associated with alterations in cerebellarmotor connectivity, as well as a decrease in vestibular connectivity, more specifically a decrease in intrinsic connectivity strength in the right insula (Fig. 3). ${ }^{60}$ This case report showed that the

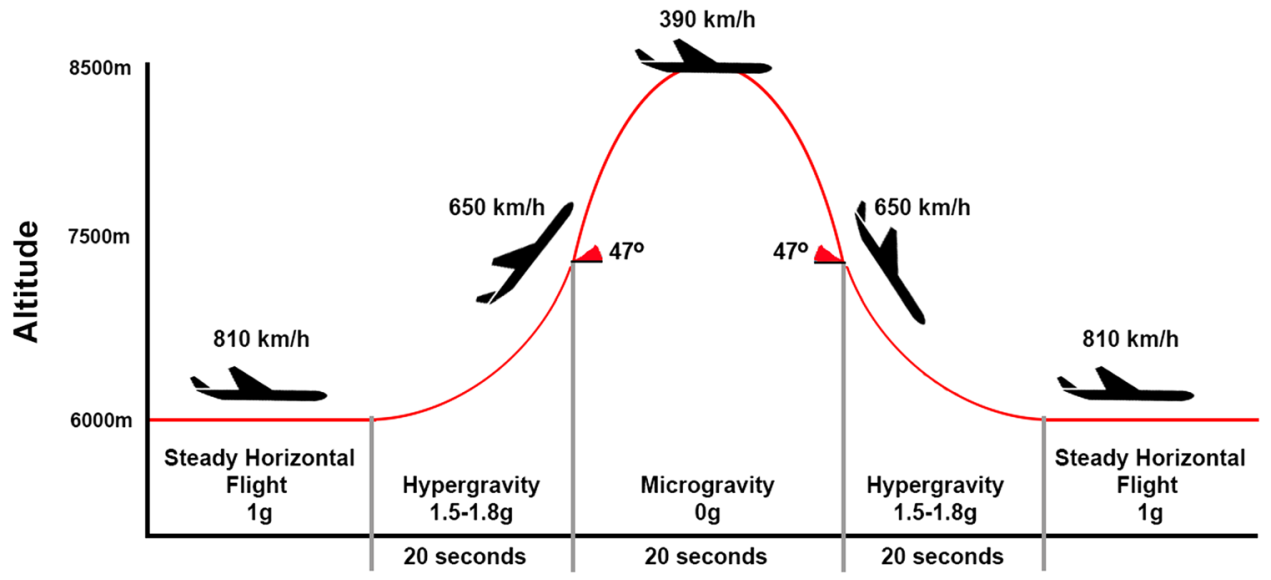

Fig. 1 Typical flight trajectory of a PF for $0 \mathrm{~g}$ parabola's 


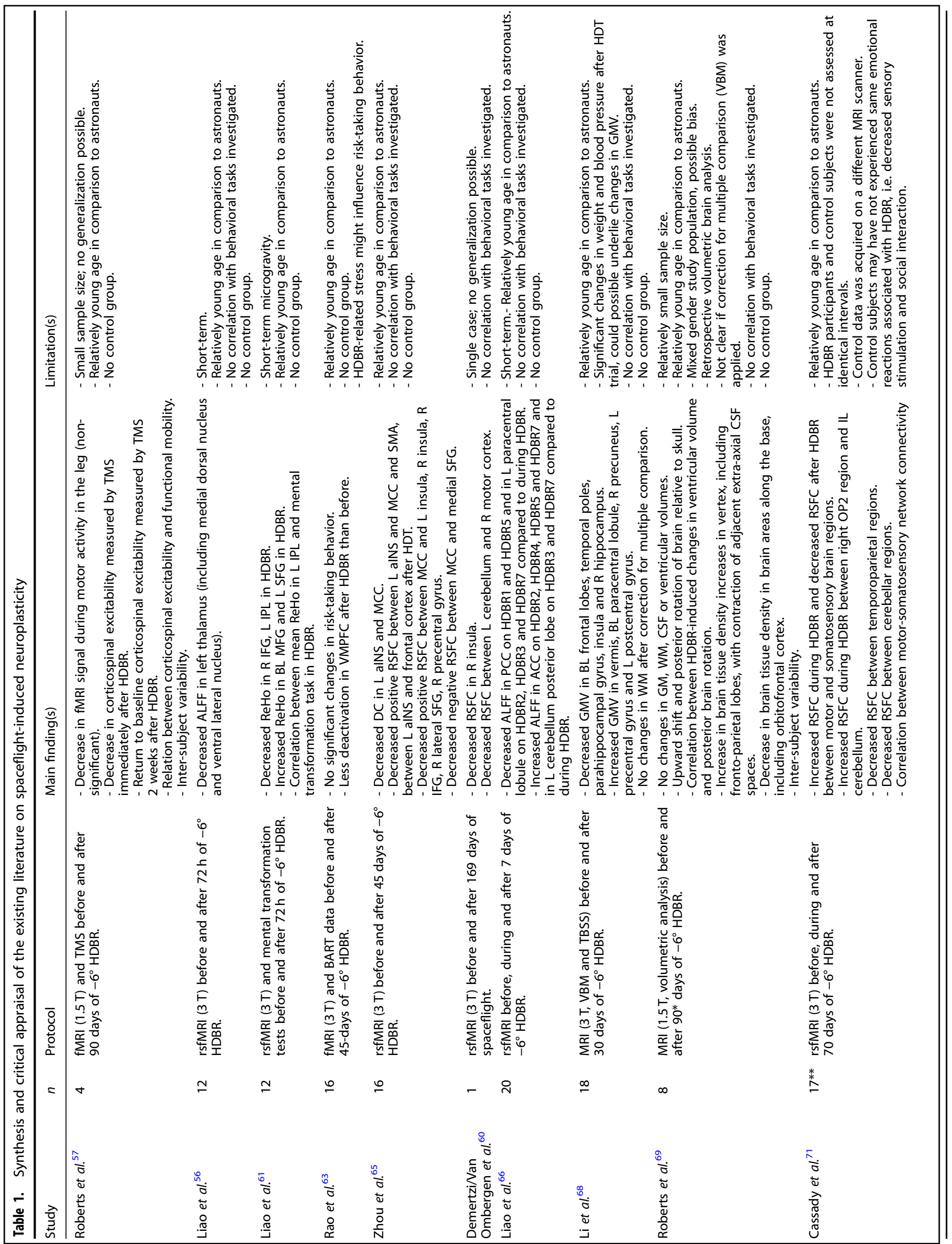




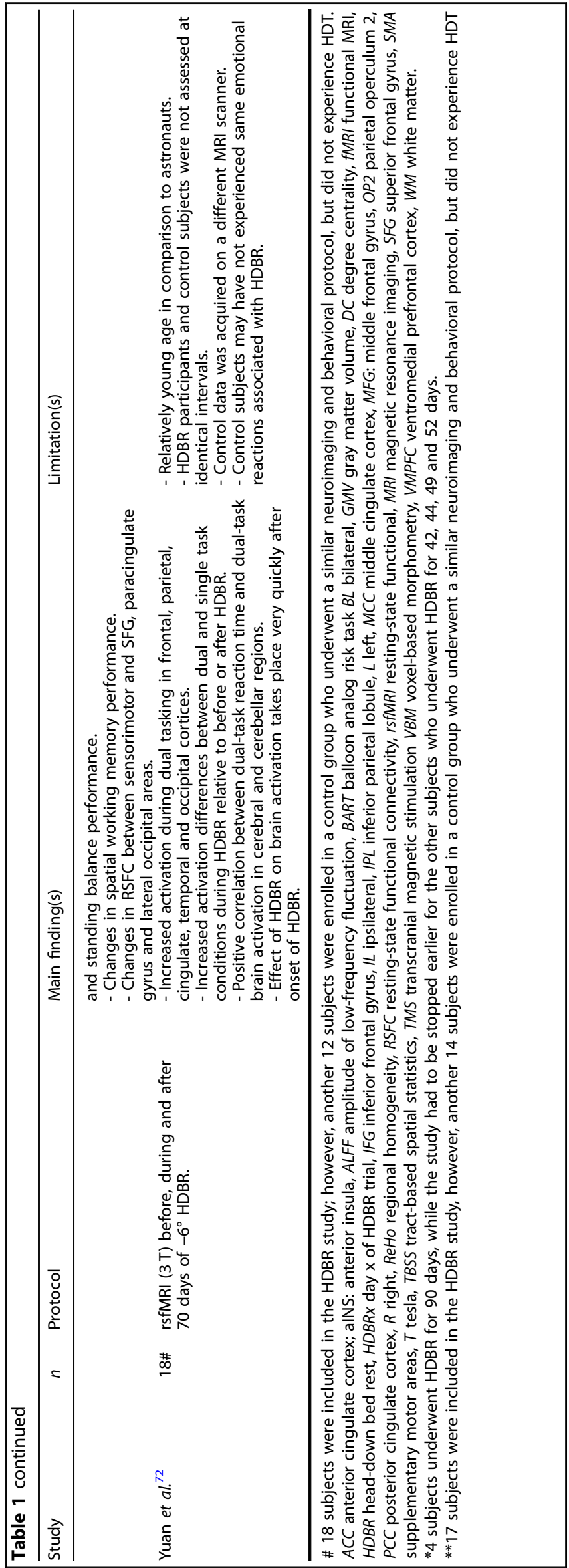

typical spaceflight-related problems such as space motion sickness, postural instability and disorientation could not solely be attributed to the peripheral end organs, i.e. the vestibular system in the inner ear, but may also have a central cortical component. However, interpretation and generalization should be very carefully made due to the anecdotal evidence. On-going longitudinal studies are aiming to extend these preliminary investigations in a larger cohort of astronauts.

Neuroplasticity and space analogs

Up until now, no MRI-based studies with dry immersion have been performed. In addition, there are no published MRI-based PF studies in humans.

Concerning HDBR, Roberts and colleagues were the first to implement a MRI-based study. ${ }^{57}$ They investigated whether simulated gravity by means of 90 days of HDBR induced changes in functional brain connectivity. In addition, they investigated corticospinal tract excitability by means of TMS. In summary, they found reduced cortical activity in the motor areas with leg representation and a decrease in corticospinal excitability after HDBR. According to the authors, these reductions in cortical motor function could underlie motor-related difficulties in astronauts. Additionally, in the post-HDBR period, they continued TMS and reported an increase in corticospinal excitability. Interestingly, they observed that the larger the increase in motor cortex excitability, the smaller the functional mobility impairment, leading them to assume that TMS could be used as a possible countermeasure against lower extremity dysfunction. Additionally, their findings could be of clinical importance, e.g. pertaining to immobilized patients or patients with lower extremity disuse.

Liao et al. initiated a HDBR study in which they investigated short-term alterations in functional connectivity. ${ }^{56}$ After $72 \mathrm{~h}$, they found decreased thalamic connectivity during resting-state, which they attributed to reduced motor control abilities and decrements in executive function in astronauts. In a follow-up study, they corroborated further on their initial results by linking them with a mental transformation test, during which the ability to perform a mental rotation strategy (i.e. mentally rotate an internal representation) is assessed. ${ }^{61}$ Interestingly, they found a correlation between intrinsic connectivity in the left inferior parietal lobe (IPL) and the mental transformation task. In addition, they found a decreased regional homogeneity (ReHo) in the IPL region, known to be involved in mental rotation strategies, ${ }^{62}$ which could explain the decrease in mental function in microgravity. Their study is interesting for the fact that they combined neuroimaging with behavioral data for the first time in regards to (simulated) spaceflight, providing an interesting insight into the link of changes in cognitive function and their underlying neural correlate.

In another fMRI study, Rao and coworkers investigated whether bed rest would influence an individual's risk-taking behavior and the underlying neural basis of this possible effect. ${ }^{63}$ They implemented the Balloon Analog Risk Task tool ${ }^{64}$ to assess risktaking. In general, they found no effect of bed rest on risk-taking behavior; however, they did find a significant deactivation of the ventromedial prefrontal cortex (VMPFC) post-HDBR when compared to before. The VMPFC is a principal component of the decision-making circuitry during risky decision-making. The finding of less deactivation of the VMPFC after HDBR is in accordance to the assumed neural adaptation process and changes in neuroplasticity after spaceflight. Furthermore, risktaking is a high-level cognitive function and therefore, plays an important role in extreme and demanding environments such as spaceflight. Therefore, their results are highly relevant, as they suggest a detrimental effect of (simulated) spaceflight on riskfull decision-making. ${ }^{63}$ 


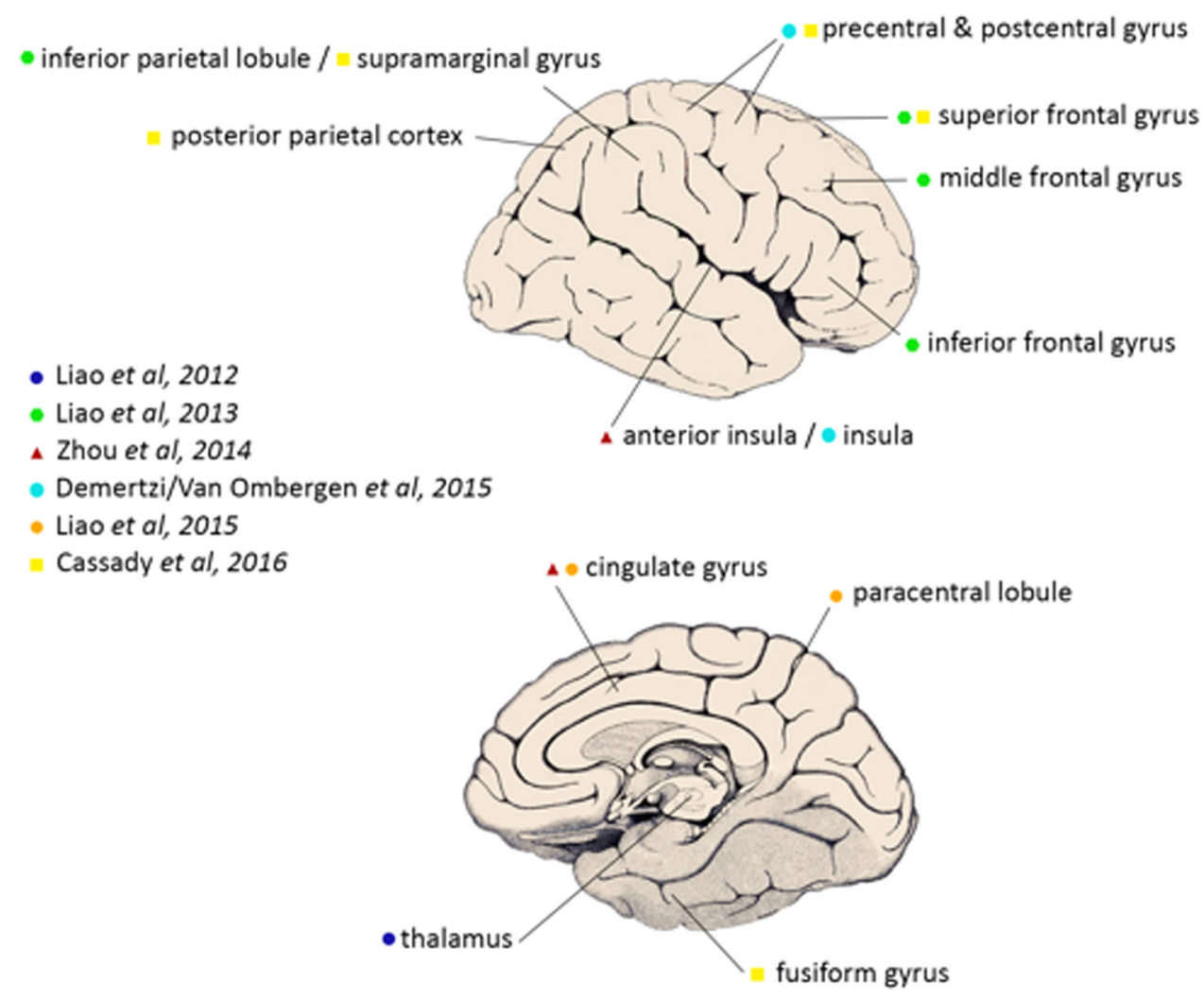

Fig. 2 Cortical and subcortical brain areas most affected by spaceflight analogs or actual spaceflight, as described in the rsfMRI studies discussed in this review (figure modified after $^{26}$, with permission, originally from ${ }^{111}$ ). For simplification, laterality of the findings was not taken into account. A more extensive description of the findings can be found in Table 1

Zhou and colleagues performed a study in which they investigated 16 healthy male individuals before and after 45 days $-6^{\circ}$ HDBR. $^{65}$ They found changes in the anterior insular and middle cingulate cortex (MCC) network, both key regions of the resting state network, that they attributed to the induced cephalic fluid shift and the concurrent increase in $\mathrm{CBF}$, intracranial pressure and oxygenated hemoglobin. In addition, the authors also suggested decreases in autonomic nervous function (i.e. sympathetic and parasympathetic) as another plausible explanation for the underlying decreases in intrinsic functional connectivity in the aINS and the MCC network. Furthermore, they postulated that the decreased anti-correlation with the superior frontal gyrus, a part of the default mode network, together with the decreased correlation within the aINS-MCC network could be the underlying neural correlates of the previously observed alterations in cognitive function occurring during microgravity. Lastly, they did not find any association with emotional state after their 45-day HDBR study. In their study, they presented a very detailed and thorough analysis of the underlying neural correlates in simulated microgravity ${ }^{65}$ Although they did not include a direct control group as such, they still validated their results by means of an independent data set acquired in healthy male volunteers, not exposed to head-down tilt bed rest, at different time points. However, like all simulated studies, it lacks the direct comparison to actual spaceflight. Spaceflight remains a unique model that even the best simulation model can't substitute and therefore, all space analog studies most likely underestimate and deviate from the complexity and multi-modal effects of human spaceflight.

Recently, Liao and colleagues published their findings from a rsfMRI study in subjects that underwent a 7-day HDBR experiment. $^{66}$ They postulated that their findings, i.e. reciprocal alterations in the posterior cingulate cortex and anterior cingulate

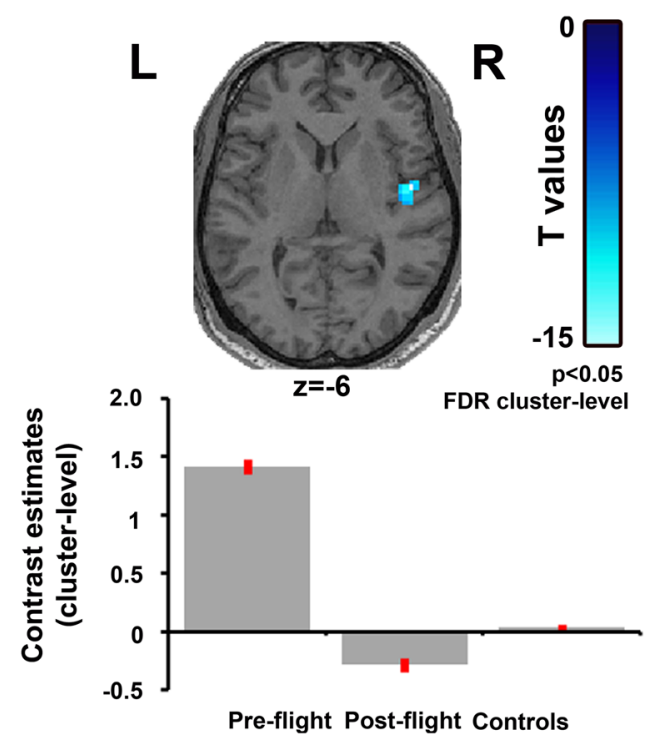

Fig. 3 The figure shows decreased connectivity strength in the right insula, a critical region of the vestibular cortex, when comparing post-flight to pre-flight in a cosmonaut. The bars represent the average connectivity strength in the respective cluster with $90 \%$ confidence interval (whiskers) for the pre-flight and post-flight scan. The statistical map is rendered on the normalized MRI scan of the cosmonaut (axial view) (from, ${ }^{60}$ used with permission)

cortex, respectively a decrease and an increase, could account for changes in the autonomic nervous system, as seen in space travelers. In addition, they found an increase in functional activity in the left cerebellar posterior lobule, which could indicate a 
compensatory role by the cerebellar posterior lobule to overcome the concurrent decline in functional connectivity in the paracentral lobule. This compensatory role of the cerebellum is postulated to be necessary to sustain adequate fine motor control and could be transferred to astronauts in a microgravity condition, where fine motor control is known to be significantly hampered. ${ }^{67}$

In another study, $\mathrm{Li}$ and co-workers demonstrated that 30 days of HDBR is associated with local GM and WM alterations. ${ }^{68}$ More specifically, they found decreases in GM volume (GMV) in the bilateral frontal lobes, temporal lobes, parahippocampal gyri, insula and hippocampus, while observing increases in GMV in the vermis, the paracentral lobules, precuneus gyrus, precentral and postcentral gyri. They related these GM changes to the decline seen in performance, locomotion, learning, memory and coordination in space travelers. Their findings should be interpreted cautiously, as their subjects experienced significant changes in weight and blood pressure after the HDBR trial, which could possibly underlie the changes in GMV.

Roberts and colleagues recently published their results from a volumetric MRI analysis in 8 subjects after long-term HDBR. ${ }^{69}$ They found several structural changes due to the simulated microgravity, with the most prominent one being the fact that the brain underwent an upward shift and posterior rotation relative to skull. Furthermore, they found a correlation between the posterior brain rotation and ventricular volume. The authors relate this to a change in CSF homeostasis and urge for further research in order to determine the exact role of this when it comes to the VIIP syndrome and its concurrent symptoms such as increased intracranial pressure, headache and visual impairment. However, a recent review doubts the feasibility of HDBR studies to investigate the effect on vision. ${ }^{70}$ Since there is no loss of tissue weight during HDBR (and any other spaceflight analog for that matter), long-duration HDBR is not a good analog for studies on vision impairment. In addition, no previous HDBR studies have reported vision impairments. ${ }^{70}$ Therefore, HDBR might not be the best model to assess VIIP syndrome and other vision-related impairments and a link with spaceflight should be made cautiously.

Very recently, a 70-day study investigated the effect of longduration HDBR on brain connectivity and behavior in 17 participants. ${ }^{71}$ A behavioral assessment as well as rsfMRI scans were conducted at 7 time points: two measurements pre-HDBR, three measurements during HDBR and two measurements postHDBR. In addition, a control group of 14 subjects was added to the study, to take into account the effects of time and practice. Interestingly, this set-up allows investigating not only the changes in brain connectivity after HDBR compared to baseline, but also the temporal changes during the HDBR. The authors reported changes in functional brain connectivity in vestibular, sensorimotor and somatosensory networks. More specifically, they observed connectivity increases during HDBR, followed by decreased connectivity after HDBR, in the motor and somatosensory cortices. The latter might imply a possible adaptive response to the HDBR environment. Therefore, the authors suggest it is plausible that motor control regions play a crucial role in this adaptation to HDBR, which is corroborated by the findings by Roberts and colleagues that 90 days of HDBR are associated with an increased motor cortex activity during foot movement immediately after HDBR and a subsequent reversal of these changes after a recovery period. ${ }^{57}$ In contrast, decreases in brain connectivity were observed between the temporoparietal regions, part of the vestibular network, and an increased functional connectivity between the right parietal operculum 2, a key region of the vestibular cortex, ${ }^{25}$ and the ipsilateral cerebellum. These findings, in conjunction to the earlier described results from ${ }^{61}$ and, ${ }^{60}$ suggest that spaceflight-related sensorimotor problems can be attributed to cortical changes at the central level. Moreover, the previously observed diminished functioning of the peripheral neurosensory organs ${ }^{22-24}$ could also be due to a central inhibition of disturbing erroneous signals coming from the vestibular organs. Furthermore, Cassady and colleagues linked their brain connectivity data with behavioral data and reported a correlation between motor-somatosensory network connectivity and standing balance performance, i.e. an individual with the greatest increase in connectivity strength between the motor and somatosensory cortices demonstrated least behavioral impairment following bed rest. This result, together with the findings from Roberts and colleagues, ${ }^{57}$ suggests that changes in body orientation and unloading, as seen in HDBR, may induce compensatory neural processes, ${ }^{71}$ a finding highly relevant for spaceflight and future space missions. Moreover, it might be the case that individual variability in neural adaptation compensates for the detrimental effects of HDBR, and spaceflight in that matter, more in some participants than in others. ${ }^{71}$

The same research group also investigated the effect of longduration HDBR on dual task performance and the underlying brain activation. ${ }^{72}$ They found increased brain activation in the frontal, parietal, cingulate and temporal cortices for dual task execution during HDBR, with a recovery to baseline levels after cessation of the HDBR. The latter implies a reduced neural efficiency in this spaceflight analog. This lower neural efficiency has been shown already during spaceflight by means of EEG recordings ${ }^{73}$ and therefore, the HDBR findings seem to be transferable to spaceflight. In addition, the aforementioned study showed that HDBR resulted in nearly immediate changes in brain activation. ${ }^{72}$ Therefore, future studies should also focus on the temporal dynamics of spaceflight-induced neuroplasticity, as indicated by these Earth-based model findings. As discussed above, preliminary spaceflight results have also found a similar effect after 6 months of spaceflight, ${ }^{60}$ but it is unknown if prolonged spaceflight has a linear or exponential effect or after which time the effects level off. A better understanding regarding the temporal characteristics of neuroplasticity is of major importance for future manned missions to the Moon and Mars.

In regards to all above-mentioned studies, it must be mentioned that HDBR induces a cephalic fluid shift that might increase CBF and thus, change the hemodynamics of the brain. ${ }^{74}$ Furthermore, also the increased intracranial pressure and oxygenated hemoglobin might alter brain hemodynamics. Therefore, this alone might already induce changes in the brain and might underlie some of the changes found in the above-mentioned studies. However, one could argue to expect more global changes in structural and functional connectivity due to fluid shifts, rather than regional specific and localized changes as described in the studies above.

Overall, we conclude, at this point of research, the HDBR analog has primarily shown alterations related to motor-related tasks (e.g. fine motor control ${ }^{66}$ ) and more advanced cognitive function such as executive function, ${ }^{56}$ mental transformation, ${ }^{61}$ spatial working memory $^{71}$ and dual tasking. ${ }^{72}$ Consequently, most studies found changes in sensorimotor, somatosensory and cognitive-related brain regions (for a full overview, see Table 1 and Fig. 2). In addition, a study in actual microgravity have additionally shown the alterations in vestibular-related cortical areas such as the insula. ${ }^{60}$ However, conclusions in regards to spaceflight need to be made carefully by both the indirect comparison of space analogs to actual spaceflight ${ }^{56,61,63,65,66,68,71,72}$ and the small sample size in some of the current studies. ${ }^{57,60,69}$

\section{GENERAL DIFFICULTIES AND LIMITATIONS OF SPACE RESEARCH}

Several HDBR studies found a large inter-subject variability. ${ }^{57,69}$ Previous spaceflight studies have already shown that inter-subject variability in space travelers is quite high, also for other physiological processes such as sensorimotor adaptation ${ }^{75,76}$ and 
vestibular and otolith deconditioning. ${ }^{77,78}$ High inter-subject variability is therefore a feature that should be kept in mind when analyzing and interpreting spaceflight studies, in particular with regards to studies on spaceflight-induced neuroplasticity. Earth-based studies have already shown that neuroplasticity is a process that is highly individually dependent and is related to several factors such as demographics (e.g. age and gender), genetic variation ${ }^{35}$ and physical activity. ${ }^{79}$

In the same line, it should be taken into account that microgravity effects on brain activation have been shown to be task dependent, as found by previous EEG studies. ${ }^{37,73}$ Therefore, the factors found to be influencing neural activation during simulated spaceflight might not only differ from actual spaceflight, they might also differ per individual and might be dependent on the specific task being executed, e.g. during task fMRI protocols.

Several other limitations are also inherent to space research with the most prominent being the small sample sizes. Up to date, approximately $150 \mathrm{crew}$ members have spent 6 months in the International Space Station (ISS), of which US astronauts [National Aeronautics and Space Administration (NASA)], Russian cosmonauts (ROSCOSMOS) and astronauts from the other space agencies (ESA, Canadian Space Agency, Japan Aerospace Exploration Agency). ${ }^{80}$ Space shuttle missions comprised more crewmembers, but the amount of time in space was not more than 2 weeks, limiting also its effects, and the space shuttle program was suspended in 2011. Unfortunately, it is very difficult to acquire data in a large group of space travelers within a reasonable time frame. Therefore, it takes quite some time for most studies to get up to an acceptable sample size, which can lead to changes in setting, equipment and team members. As an example with regards to MRI-based studies, longitudinal studies could lead to variability in MRI acquisition parameters between scans and therefore, potentially confound observed changes. ${ }^{81}$ In addition, MRI acquisition technology changes rapidly and state-of-the-art pre-processing and statistical analysis techniques develop at a fast rate. ${ }^{82}$ Therefore, a longitudinal study over a long period of time could lead to the fact that out-dated techniques are being used for consistency among measurements.

In addition, also due to logistic restrictions, it is very difficult and often impossible to assess space travelers in the first few hours or days after returning from space due to restrictions in the schedule of astronauts. For neuroplasticity measurements, it could be possible that there is a critical time frame within which changes are detectable by means of MRI measurements. Also, when assessment can only take place a couple of days after returning to Earth, one is not only measuring the spaceflight-induced changes, but also the changes taking place due to re-adaptation back on Earth. ${ }^{60}$ This can hamper the detection of more subtle changes or can even counteract these processes in some cases. Especially in the framework of neuroplasticity, it is known that changes can take place on a very short period of time, e.g. alterations in WM structure can already take place after $90 \mathrm{~min}$ of a spatial learning task. $^{83,84}$ Therefore, neuroplasticity assessments must be made at well-considered and repeated time points. This is also relevant for studies in which a spaceflight alternative is implemented, however, in general the logistic and scheduling restrictions are easier to overcome or adjust compared to spaceflight.

When focussing on neuroplasticity measured by MRI only, we can only assess the human brain before and after spaceflight. Due to loads of technical, logistic and payload restrictions, there is no possibility to take an MRI-scanner into space or to the ISS. Therefore, it is not possible to assess neuroplastic events, probed by MRI-techniques, during spaceflight, although this would lead to very interesting insights. However, we could complement beforeafter MRI assessment with more portable neuroimaging techniques on board such as EEG, TMS or near-infrared spectroscopy (NIRS) and by correlating post-spaceflight changes as measured by MRI with behavioral measurements taken on board.
Another complicating factor is the specific demographic profile of space travelers. In general, there is a well-known majority of male space travelers compared to female space travelers with a ratio of roughly 9 to 1 respectively ${ }^{85}$ (In addition, the mean age of astronauts on their first-time flight to space is slightly different for males and females: 44.5 years vs. 42.5 years ${ }^{80}$ ). It is therefore important that Earth-based space analogs take this into account in order to resemble the demographic profile as much as possible. It is also known that gender can have an impact on the adaptation of several physiological systems to spaceflight. ${ }^{80,86-88}$ Previous studies on neuroplasticity showed that gender and age could influence the degree and extent of neuroplasticity. The menstrual cycle for example can impact on structural and functional neural adaptations. ${ }^{89}$ Therefore, if space analog studies on neuroplasticity want to transfer their findings to make assumptions or conclusions on spaceflight-induced neuroplasticity, they should match age and gender features as much as possible.

\section{IMPLICATIONS FOR COUNTERMEASURES AND NEUROIMAGING IN SPACEFLIGHT-RELATED STUDIES}

We should aim to accurately determine and map the effect of changes in brain structure and function on the motor, vestibular and cognitive system in order to make long-duration missions (e.g. during several years) feasible and possible. In a second phase, suitable countermeasures should be determined and applied. The ability to perform landing and post-landing tasks (e.g. on Mars) may be hampered by impaired motor control, movement and motor coordination. This could encumber crew performance, crew safety and may even compromise the mission. Furthermore, higher cognitive tasks (e.g. working memory, risk-taking and dualtasking) might be influenced, possibly leading to unacceptable risks and hazards in spaceflight, where there is a high working load and stress situations might occur frequently and/or suddenly.

\section{Countermeasures}

Recently, the idea of motor imagery (MI), an experimental paradigm already widely used in sports, has been proposed as an inexpensive and rather simple approach to prepare space travelers for the absence of gravity they will encounter. ${ }^{90} \mathrm{MI}$ is a process during which a specific and pre-decided action is internally reproduced in working memory, from a first-person perspective, without any overt motor output. ${ }^{91}$ It typically includes multiple sensory modalities, e.g., mentally visualizing a specific motor task and mentally feeling muscle contractions. ${ }^{92}$ Imagined and executed movements have been shown to have the same vividness and temporal structure ${ }^{93,94}$ and in addition, it has been proven that $\mathrm{Ml}$ activates similar brain regions as is the case with executed movements, e.g. primary and secondary motor cortices, posterior parietal cortex, basal ganglia and the cerebellum. ${ }^{95,96}$ This kind of mental practice could be applied to prepare astronauts to the sudden absence of gravity and to the readaptation phase when coming back to Earth. ${ }^{90}$

Additionally, the study from Roberts and colleagues showed TMS to be a possible countermeasure. ${ }^{57}$ TMS is portable and therefore, possible to be implemented in space. The authors suggest TMS to become part of a countermeasure regime for astronauts on long-duration space missions to counteract lower extremity dysfunction, ${ }^{57}$ e.g. prior to operations on a planetary surface as might be the case for interplanetary missions. Another topic well discussed among space researchers, is artificial gravity as a countermeasure. By introducing continuous or intermittent exposure to artificial gravity (or some sort of gravitational levels), the adaptation to e.g. Martian gravity or re-adaptation to Earth's gravity might be facilitated. ${ }^{97}$ For example, this could be done by introducing a centrifuge on board. ${ }^{98}$ By doing so, the physiological deconditioning, as seen after exposure to weightlessness, could 
be counteracted. Undoubtedly, this will also affect the human brain and the underlying neural adaptation to spaceflight. Future studies should investigate to what extent artificial gravity (by means of centrifuge or otherwise) plays a possible role in neuroplasticity.

\section{Neuroimaging}

In regards to neuroimaging specifically, the current literature suffers from the fact that all studies are using different acquisition, data pre-processing and statistical analysis techniques, as well as a different set-up for their experiments. Furthermore, several different analysis techniques such as for example BOLD connectivity measures using hypothesis-driven seed-voxel analyses or data-driven independent component analyses; amplitude of lowfrequency fluctuation or ReHo measures have been used, adding to the difficulty to compare different studies with each other. However, since these are analysis happening at the postprocessing level, they allow for re-analysis and comparison with more widely used connectivity approaches.

In addition, and this holds true for the majority of neuroimaging techniques: all of the above are indirect measures of synaptic neural activity. ${ }^{48}$ For example, changes in brain volume found with volumetric analyses tools do not allow the possibility to make a conclusion on changes at the cytoarchitectonial level. Moreover, changes in GM (and WM to some extent) could be the result of changes in neuropil, changes in neuronal size, dendritic and axonal adaptations, as well as be related to folding or the development of thicker GM. ${ }^{99}$ In addition to the complexity of the precise origin of GM changes, various factors are known to have an impact on brain morphology and may therefore cause brain volume changes. Also, the difference between short-term and long-term exposure to (simulated) microgravity is of course very relevant, but this intrinsically hampers the comparability among studies. Data sharing and weighted meta-analyses could be proposed for future analyses.

Cognitive changes due to spaceflight might be associated with metabolic changes, even before the occurrence of "clinical" symptoms and this relation should be further examined by means of state-of-the-art techniques such as PET scans ${ }^{32}$ or MRI spectroscopy. These techniques could probe changes in neurotransmitter systems e.g. dopamine receptor activity. Based on findings from earlier animal studies related to spaceflight, it is hypothesized to find changes in humans as well. ${ }^{30,100}$ Earth-based studies have shown that changes in neurotransmitters have major implications for attention, ${ }^{101}$ (long-term) memory, ${ }^{102}$ arousal ${ }^{103}$ and motor activity. ${ }^{104}$ Determining neurotransmitter and hormonal imbalance in space travelers is therefore important to get fundamental insight into how the central neural system adapt to microgravity and in addition, to get insight into the relation between these alterations and behavioral processes.

In relation to spaceflight, it is needed to determine the temporal profile and longevity of neuroplastic changes and correlate these with the temporal profile of the (re-)adaptation process and possible detrimental changes. Therefore, in vivo neuroimaging techniques such as MRI and EEG are crucial as they allow mapping structural, functional and metabolic events in the human brain in relation to microgravity and spaceflight. Gaining insight into the dynamic properties of the human brain over time could also help in the development and application of countermeasures as well as help to determine when or how long they should be applied. ${ }^{105}$

In preparing for (very) long-duration interplanetary missions, it is important to determine the impact of changes in brain structure and function on sensori-motor, higher cognitive and psychological capacities of space travelers, since brain alterations might interfere with the decline in brain volume and functional reorganization and connectivity as seen in a normal ageing population. ${ }^{106-108}$ If this is the case, this might potentially lead to accelerated cerebral aging effects and concurrent accelerated decline, e.g. sensory impairment, motor slowing, memory problems, deficits in attention and processing speed and anxio-depressive disorders (e.g., 112,113).

In general, simultaneous and independent multimodal neuroimaging is pivotal to acquire a still lacking understanding of functional and structural brain processes in relation to human spaceflight. The combination of different complementary electrophysiological and neuroimaging techniques should be used to acquire non-redundant information, e.g. structural, functional and metabolic MRI pre and post spaceflight combined with highdensity EEG, TMS and/or NIRS. Not only would this give a more complete insight into spaceflight-induced neuroplasticity, but also would the simultaneous use of different techniques overcome limitations inherent to one single technique. An example of this is combining EEG and MRI for a more efficient assessment of the temporal dynamics and spatial information of the underlying neural processes taking place, i.e. to improve and optimize spatiotemporal resolution. ${ }^{109}$

Another feasible approach would be to validate several motorrelated and cognitive tasks on Earth by means of fMRI, which would then allow making predictions on brain alterations when performing these tasks inflight in the ISS for example. Illustrations are tests for sensorimotor skills, attention, working memory, spatial orientation, etc. These can be easily done on board of the ISS since they are portable, non-expensive and non-time consuming. A good example hereof is the "Cognition" test battery that is currently being implemented by NASA. ${ }^{110}$

\section{CONCLUSION AND FUTURE PERSPECTIVES}

In conclusion, despite the discussed limitations of the current literature regarding heterogeneous experimental set-ups and the lack of comparability of findings among studies, some trends have been witnessed. The cerebellum, cortical motor areas and vestibular-related pathways seem to be critically involved across different studies, indicating that these brain regions are indeed affected by real and simulated spaceflight. These changes reflect most likely an underlying neural component of the common detrimental changes observed in space travelers such as problems with sensorimotor control and motor coordination, space motion sickness and a hampered otolith and vestibulo-autonomic functioning.

Currently, there is paucity in the knowledge of the effect of microgravity on the human brain and more extensive research is therefore highly needed to increase and add more insight into this matter. The relationship between spaceflight-related physiological and neuro-psychological problems and alterations in brain structure or function should be investigated. Elaborating on the understanding of how the brain reacts to and behaves in spaceflight is a crucial step in the development of more adequate countermeasures against the detrimental changes often seen in space travelers. Assessing space travelers by means of validated and standardized multimodal neuroimaging protocols will help establish a more precise picture of functional, structural and biochemical brain alterations associated with spaceflight. Hereto, it could be of interest to develop a protocol comprising of the minimum of tests that should be performed to optimize merging among studies as much as possible. Within the framework of the space agencies, an international multi-disciplinary task-force or topical team should be established to set-up such a list.

Extending this knowledge is pivotal to guarantee the safety and efficiency of future space missions, such as interplanetary missions to Mars and the development of permanent space habitats. Furthermore, the development, safety and success of commercial space tourism are dependent on how a less-trained human being reacts to this short-term exposure to microgravity, including possible alterations at the level of the brain. Lastly, the acquired 
insights in this unique population of space travelers have direct and indirect clinical impacts and could be transferred to multiple neurological and psychiatric diseases and pathologies on Earth such as patients suffering from neurodegenerative disorders, vestibular problems and motor immobilization.

\section{ACKNOWLEDGEMENTS}

This work was supported by the European Space Agency (ESA) and BELSPO Prodex, the University and University Hospital of Antwerp and the Research Foundation Flanders (FWO Vlaanderen). AVO is a Research Fellow of the Research Foundation Flanders (Belgium, FWO Vlaanderen). SL is Research Director at the Fonds National de la Recherche Scientifique (FNRS). AVO received a 2016-2017 Amelia Earhart fellowship from Zonta International. The authors would like to thank Jeroen De Coninck for the help with the Fig. 2.

\section{COMPETING INTERESTS}

The authors declare that they have no conflict of interest.

\section{REFERENCES}

1. Clément G., Reschke M. Neuroscience in Space. p 322 (Springer, 2008).

2. Kanas N., Manzey D. Space Psychology and Psychiatry 2nd edn p 240 (Springer, 2008).

3. Williams, D., Kuipers, A., Mukai, C. \& Thirsk, R. Acclimation during space flight: effects on human physiology. Can. Med. Assoc. J. 180, 1317-1323, http:// www.pubmedcentral.nih.gov/articlerender.fcgi?artid=2696527\&tool=pmcentrez \&rendertype=abstract (2009).

4. Kramer, L. A., Sargsyan, A. E., Hasan, K. M., Polk, J. D. \& Hamilton, D. R. Orbital and intracranial effects of microgravity: findings at 3-T MR imaging. Radiology 263, 819-827, http://www.scopus.com/inward/record.url?eid=2-s2.0-84862500034 \&partnerID=tZOtx3y1 (2012).

5. Riascos, R. et al. Novel finding of optic nerve central T2 hypointensity utilizing 3 Tesla MR imaging. Neuroradiol. J. 28, 133-136 (2015).

6. Schneider, S. et al. Changes in cerebral oxygenation during parabolic flight. Eur. J. Appl. Physiol. 113, 1617-1623 (2013).

7. Taylor, C. R. et al. Spaceflight-induced alterations in cerebral artery vasoconstrictor, mechanical, and structural properties: implications for elevated cerebral perfusion and intracranial pressure. FASEB J. 27, 2282-2292 (2013).

8. Blaber, A. P., Goswami, N., Bondar, R. L. \& Kassam, M. S. Impairment of cerebral blood flow regulation in astronauts with orthostatic intolerance after flight. Stroke 42, 1844-1850 (2011).

9. Nelson, E., Mulugeta, L. \& Myers, J. Microgravity-induced fluid shift and ophthalmic changes. Life 4, 621-665 (2014).

10. Aubert, A. E., Beckers, F. \& Verheyden, B. Cardiovascular function and basics of physiology in microgravity. Acta Cardiol. 60, 129-151 (2005).

11. Sonnenfeld, G. \& Shearer, W. T. Immune function during space flight. Nutrition. 18, 899-903 (2002)

12. Nickerson, C. A., Ott, C. M., Wilson, J. W., Ramamurthy, R. \& Pierson, D. L. Microbial responses to microgravity and other low-shear environments. Microbiol. Mol. Biol. Rev. 68, 345-361, http://www.pubmedcentral.nih.gov/ articlerender.fcgi?artid=419922\&tool=pmcentrez\&rendertype=abstract (2004).

13. Fitts, R. H., Riley, D. R. \& Widrick, J. J. Functional and structural adaptations of skeletal muscle to microgravity. J. Exp. Biol. 204, 3201-3208 (2001).

14. Stein, T. P. Weight, muscle and bone loss during space flight: another perspective. Eur. J. Appl. Physiol. 113, 2171-2181 (2013).

15. Fowler, B., Comfort, D. \& Bock, O. A review of cognitive and perceptual-motor performance in space. Aviat. Space Environ. Med. 71, A66-A68, http://www.ncbi. nlm.nih.gov/pubmed/10993312 (2000).

16. Clément, G. \& Ngo-Anh, J. T. Space physiology II: adaptation of the central nervous system to space flight-past, current, and future studies. Eur. J. Appl. Physiol. 113, 1655-1672 (2013).

17. Clément, G., Reschke, M. \& Wood, S. Neurovestibular and sensorimotor studies in space and Earth benefits. Curr. Pharm. Biotechnol. 6, 267-283, http://www. ncbi.nlm.nih.gov/pubmed/16101466 (2005).

18. Lackner, J. R. \& DiZio, P. Space motion sickness. Exp. Brain Res. 175, 377-399 (2006).

19. Strangman, G. E., Sipes, W. \& Beven, G. Human cognitive performance in spaceflight and analogue environments. Aviat. Space Environ. Med. 85, 1033-1048 (2014).
20. Kozlovskaya, I., Barmin, V., Stepantsov, V. \& Kharitonov, N. Results of studies of motor functions in long-term space flights. Physiologist 33, S1-S3 (1990).

21. Manzey D. Human missions to Mars: new psychological challenges and research issues. Acta Astronaut. 55(3-9), 781-790 (2004)

22. Moore, S. et al. No ocular and perceptual responses to linear acceleration in microgravity: alterations in otolith function on the COSMOS and Neurolab flights. J. Vestib. Res. 12, 377-393 (2003).

23. Kornilova, L., Naumov, I., Azarov, K. \& Sagalovitch, V. Gaze control and vestibularcervical-ocular responses after prolonged exposure to microgravity. Aviat. Sp. Env. Med. 83, 1123-1134 (2012).

24. Hallgren E., et al. Decreased otolith-mediated vestibular response in 25 astronauts induced by long duration spaceflight. J Neurophysiol. http://www.ncbi. nlm.nih.gov/pubmed/27009158 (2016).

25. Zu Eulenburg, P., Caspers, S., Roski, C. \& Eickhoff, S. B. Meta-analytical definition and functional connectivity of the human vestibular cortex. Neuroimage 60, 162-169 (2012).

26. Lopez, C. \& Blanke, O. The thalamocortical vestibular system in animals and humans. Brain Res. Rev. 67, 119-146 (2011).

27. Libet, B., Alberts, W. W., Wright, E. W. \& Feinstein, B. Responses of human somatosensory cortex to stimuli below threshold for conscious sensation. Science 158, 1597-1600 (1967).

28. De La Torre, G. Cognitive neuroscience in space. Life. 4, 281-294 (2014).

29. Ivry, R. B., Keele, S. W. \& Diener, H. C. Dissociation of the lateral and medial cerebellum in movement timing and movement execution. Exp. Brain. Res. 73, 167-180 (1988).

30. Newberg, A. B. Changes in the central nervous system and their clinical correlates during long-term spaceflight. Aviat. Space. Environ. Med. 65, 562-572 (1994).

31. Correia, M. J. Neuronal plasticity: adaptation and readaptation to the environment of space. Brain. Res. Rev. 28, 61-65, http://www.ncbi.nlm.nih.gov/pubmed/ 9795137 (1998).

32. Newberg, A. B. \& Alavi, A. Changes in the central nervous system during longduration space flight: implications for neuro-imaging. Adv. Space. Res. 22, 185-196 (1998).

33. Kosik, K. Neurolab: learning how the nervous system adapts to microgravity. Neurosci. News. 5, 36-38 (1998).

34. Slenzka, K. Neuroplasticity changes during space flight. Adv. Sp. Res. 31, 1595-1604 (2003).

35. Pearson-Fuhrhop, K. M. \& Cramer, S. C. Genetic influences on neural plasticity. PM. R. 2, S227-S240, doi:10.1016/j.pmrj.2010.09.011 (2010).

36. Marušič, U., Meeusen, R., Pišot, R. \& Kavcic, V. The brain in micro- and hypergravity: the effects of changing gravity on the brain electrocortical activity. Eur. J. Sport Sci. doi:10.1080/17461391.2014.908959 (2014).

37. Cheron, G. et al. Effect of gravity on human spontaneous $10-\mathrm{Hz}$ electroencephalographic oscillations during the arrest reaction. Brain Res. 1121, 104-116 (2006).

38. Schneider, S. et al. What happens to the brain in weightlessness? A first approach by EEG tomography. Neuroimage 42, 1316-1323 (2008).

39. Babiloni, C., Pizzella, V., Del Gratta, C., Ferretti, A. \& Romani, G. L. Fundamentals of electroencefalography, magnetoencefalography, and functional magnetic resonance imaging. Int. Rev. Neurobiol. 86, 67-80, http://www.ncbi.nlm.nih.gov/ pubmed/19607991 (2009).

40. Ashburner, J. \& Friston, K. J. Voxel-based morphometry-the methods. Neuroimage 11, 805-821 (2000).

41. Basser, P. J., Mattiello, J. \& LeBihan, D. MR diffusion tensor spectroscopy and imaging. Biophys. J. 66, 259-267 (1994).

42. Basser, P. J. \& Pierpaoli, C. Microstructural and physiological features of tissues elucidated by quantitative-diffusion-tensor MRI. J. Magn. Reson. B. 111, 209-219 (1996).

43. Basser, P., Pajevic, S., Pierpaoli, C., Duda, J. \& Aldroubi, A. In vivo fiber tractography using DT-MRI data. Magn. Reson. Med. 44, 625-632 (2000).

44. Mori, S., Crain, B. J., Chacko, V. P. \& van Zijl, P. C. Three-dimensional tracking of axonal projections in the brain by magnetic resonance imaging. Ann. Neurol. 45, 265-269, http://www.ncbi.nlm.nih.gov/pubmed/9989633 (1999).

45. Smith, S. M. et al. Tract-based spatial statistics: voxelwise analysis of multisubject diffusion data. Neuroimage 31, 1487-1505 (2006).

46. Duyn, J. H., Moonen, C. T., van Yperen, G. H., de Boer, R. W. \& Luyten, P. R. Inflow versus deoxyhemoglobin effects in BOLD functional MRI using gradient echoes at 1.5 T. NMR Biomed. 7, 83-88, http://www.ncbi.nlm.nih.gov/pubmed/8068530 (1994).

47. Forster, B. B. et al. Functional magnetic resonance imaging: the basics of blood-oxygen-level dependent (BOLD) imaging. Can Assoc. Radiol. J. 49, 320-329, http://www.ncbi.nlm.nih.gov/entrez/query.fcgi?cmd=Retrieve\&db=PubMed\&dopt= Citation\&list_uids=9803232 (1998). 
48. Hamaide, J., De Groof, G. \& Van der Linden, A. Neuroplasticity and MRI: a perfect match. Neuroimage 131, 13-28 (2016).

49. Bestmann, S. The physiological basis of transcranial magnetic stimulation. Trends Cogn. Sci. 12, 81-83, http://www.ncbi.nlm.nih.gov/pubmed/18243042 (2008).

50. Ferreri, F. \& Rossini, P. M. TMS and TMS-EEG techniques in the study of the excitability, connectivity, and plasticity of the human motor cortex. Rev. Neurosci. 24, 431-442 (2013).

51. Davey, N. J. et al. Human corticospinal excitability in microgravity and hypergravity during parabolic flight. Aviat. Space Environ. Med. 75, 359-363, http:// www.ncbi.nlm.nih.gov/pubmed/15086127 (2004).

52. Navasiolava, N. M. et al. Long-term dry immersion: Review and prospects. Eur. J. Appl. Physiol. 111, 1235-1260 (2011).

53. Moore, S. T., MacDougall, H. G. \& Paloski, W. H. Effects of head-down bed rest and artificial gravity on spatial orientation. Exp. brain. Res. 204, 617-622, http:// www.ncbi.nlm.nih.gov/pubmed/20535455 (2010).

54. Pavy-Le Traon, A., Heer, M., Narici, M. V., Rittweger, J. \& Vernikos, J. From space to Earth: advances in human physiology from 20 years of bed rest studies (1986-2006). Eur. J. Appl. Physiol. 101, 143-194 (2007).

55. De La Torre, G. G. et al. Future perspectives on space psychology: recommendations on psychosocial and neurobehavioural aspects of human spaceflight. Acta Astronaut. 81, 587-599 (2012).

56. Liao, Y. et al. Altered baseline brain activity with $72 \mathrm{~h}$ of simulated microgravity -initial evidence from resting-state fMRI. PLoS One 7, 1-6 (2012).

57. Roberts, D. R. et al. Cerebral cortex plasticity after 90 days of bed rest: data from TMS and fMRI. Aviat. Space Environ. Med. 81, 30-40 (2010).

58. Karmali, F. \& Shelhamer, M. The dynamics of parabolic flight: flight characteristics and passenger percepts. Acta Astronaut. 63, 594-602, http://www.pub medcentral.nih.gov/articlerender.fcgi?artid=2598414\&tool=pmcentrez\&render type=abstract (2008).

59. Jacubowski, A. et al. The impact of long-term confinement and exercise on central and peripheral stress markers. Physiol. Behav. 152, 106-111 (2015).

60. Demertzi, A. et al. Cortical reorganization in an astronaut's brain after long duration spaceflight. Brain. Struct. Funct. 221, 2873-2876 (2016).

61. Liao, Y., Miao, D., Huan, Y., Yin, H., Xi, Y. \& Liu, X. Altered regional homogeneity with short-term simulated microgravity and its relationship with changed performance in mental transformation. PLoS One 8, e64931, http://www.pub medcentral.nih.gov/articlerender.fcgi?artid=3670926\&tool=pmcentrez\&render type $=$ abstract (2013).

62. Vingerhoets, G., de Lange, F. P., Vandemaele, P., Deblaere, K. \& Achten, E. Motor imagery in mental rotation: an fMRI study. Neuroimage 17, 1623-1633, http://www.sciencedirect.com/science/article/pii/S1053811902912905\nhttp://li nkinghub.elsevier.com/retrieve/pii/S1053811902912905 (2002).

63. Rao, L.-L. et al. Decreasing ventromedial prefrontal cortex deactivation in risky decision making after simulated microgravity: effects of $-6^{\circ}$ head-down tilt bed rest. Front. Behav. Neurosci. 8, 187, http://www.pubmedcentral.nih.gov/ articlerender.fcgi?artid=4034329\&tool=pmcentrez\&rendertype=abstract (2014).

64. Lejuez, C. W. et al. Evaluation of a behavioral measure of risk taking: the Balloon Analogue Risk Task (BART). J. Exp. Psychol. Appl. 8, 75-84 (2002).

65. Zhou, Y. et al. Disrutpted resting-state functional architecture of the brain after 45-day simulated microgravity. Front. Behav. Neurosci. 8, 200, http:// www.pubmedcentral.nih.gov/articlerender.fcgi?artid=4046318\&tool=pmcentrez \&rendertype=abstract (2014).

66. Liao Y., et al. The time course of altered brain activity during 7-day simulated microgravity. Front Behav Neurosci. 2015

67. Lackner, J. R. \& DiZio, P. Motor function in microgravity: movement in weightlessness. Curr. Opin. Neurobiol. 6, 744-750, http://ac.els-cdn.com/ S0959438896800237/1-s2.0-S0959438896800237-main.pdf? tid=79b0be518b88 e876c5a353de5d6bc224\&acdnat=1341312016_a5fa00f86cd1483ce32b1b2c52b $6 d 966$ (1996).

68. Li K., et al. Effect of simulated microgravity on human brain gray matter and white matter - evidence from MRI. PLoS One 2015.

69. Roberts, D. et al. Structural brain changes following long-term $6^{\circ}$ head-down tilt bed rest as an analog for spaceflight. Am. J. Neuroradiol. 36, 2048-2054 (2015)

70. Hargens, A. R. \& Vico, L. Long-duration bed rest as an analog to microgravity. J. Appl. Physiol. 120, 891-903 (2016).

71. Cassady, K. et al. Effects of a spaceflight analog environment on brain connectivity and behavior. Neuroimage 141, 18-30 (2016).

72. Yuan, P. et al. Increased brain activation for dual tasking with 70-days headdown bed rest. Front. Syst. Neurosci. 10, 71 (2016).

73. Cheron $\mathrm{G}$, et al. Gravity influences top-down signals in visual processing. PLoS One (2014).

74. Liang, X., Zou, Q., He, Y. \& Yang, Y. Coupling of functional connectivity and regional cerebral blood flow reveals a physiological bias for network hubs of the human brain. Proc. Natl. Acad. Sci. USA 110, 1929-1934 (2013).
75. Wood, S. J., Loehr, Ja \& Guilliams, M. E. Sensorimotor reconditioning during and after spaceflight. NeuroRehabilitation 29, 185-195, http://www.ncbi.nlm.nih.gov/ pubmed/22027081 (2011).

76. Seidler, R. D., Mulavara, A. P., Bloomberg, J. J. \& Peters, B. T. Individual predictors of sensorimotor adaptability. Front. Syst. Neurosci. 9, 1-10, http://journal. frontiersin.org/article/10.3389/fnsys.2015.00100/abstract (2015).

77. Buytaert, K. I. et al. Validation of centrifugation as a countermeasure for otolith deconditioning during spaceflight: preliminary data of the ESA SPIN study. J. Vestib. Res. 23, 23-31, http://www.ncbi.nlm.nih.gov/pubmed/23549052 (2013).

78. Hallgren E., et al. Dysfunctional vestibular system causes a blood pressure drop in astronauts returning from space. Sci. Rep. 5, (2015).

79. Bherer, L. Cognitive plasticity in older adults: effects of cognitive training and physical exercise. Ann. N. Y. Acad. Sci. 1337, 1-6, http://www.ncbi.nlm.nih.gov/ pubmed/25773610 (2015).

80. Goel, N. et al. Effects of sex and gender on adaptation to space: behavioral health. J. Women's Heal. 23, 975-986, http://online.liebertpub.com/doi/abs/ 10.1089/jwh.2014.4911 \nhttp://online.liebertpub.com/doi/pdfplus/10.1089/jwh. 2014.4911 (2014).

81. Chua, A. et al. Handling changes in MRI acquisition parameters in modeling whole brain lesion volume and atrophy data in multiple sclerosis subjects: comparison of linear mixed-effect models. Neurolmage: Clin. 8 , 606-610 (2015)

82. Ai, T. et al. A historical overview of magnetic resonance imaging, focusing on technological innovations. Invest. Radiol. 47, 1 (2012).

83. Tavor, I., Hofstetter, S. \& Assaf, Y. Micro-structural assessment of short term plasticity dynamics. Neuroimage 81, 1-7 (2013).

84. Sagi, Y. et al. Learning in the fast lane: new insights into neuroplasticity. Neuron 73, 1195-1203 (2012)

85. Mark, S. et al. The impact of sex and gender on adaptation to space: executive summary. J. Womens Health 23, 941-947 (2014).

86. Reschke, M. F. et al. Effects of sex and gender on adaptation to space: neurosensory systems. J Womens Health 23, 959-962, http://www.ncbi.nlm.nih.gov/ pubmed/25401941 (2014).

87. Ploutz-Snyder, L. et al. Effects of sex and gender on adaptation to space: musculoskeletal health. J. women's Heal. 23, 963-966, http://www.ncbi.nlm.nih. gov/pubmed/25401942 (2014).

88. Kennedy, A. R. et al. Effects of sex and gender on adaptation to space: immune system. J. Womens Health. 23, 956-958, http://www.ncbi.nlm.nih.gov/pubmed/ 25401940 (2014).

89. Barth, C., Villringer, A. \& Sacher, J. Sex hormones affect neurotransmitters and shape the adult female brain during hormonal transition periods. Front. Neurosci. 9, 37, http://www.pubmedcentral.nih.gov/articlerender.fcgi? artid=4335177\&tool=pmcentrez\&rendertype=abstract (2015).

90. Bock, O., Schott, N. \& Papaxanthis, C. Motor imagery: lessons learned in movement science might be applicable for spaceflight. Front. Syst. Neurosci. 18, 75 (2015).

91. Decety, Grèzes Neural mechanisms subserving the perception of human actions. Trends Cogn. Sci. 3, 172-178, http://www.ncbi.nlm.nih.gov/pubmed/ 10322473 (1999).

92. Weinberg R. Does imagery work? effects on performance and mental skills. J. Imag. Res. Sport Phys. Act. 3, (2008).

93. Guillot, A. \& Collet, C. Construction of the motor imagery integrative model in sport: a review and theoretical investigation of motor imagery use. Int. Rev. Sport. Exerc. Psychol. 1, 31-44 (2008).

94. Papaxanthis C., Paizis C., White O., Pozzo T., Stucchi N. The relation between geometry and time in mental actions. PLoS One 7, (2012).

95. Jeannerod, $M$. The representing brain: neural correlates of motor intention and imagery. Behav. Brain. Sci. 17, 187 (1994).

96. Munzert J., Zentgraf K. Motor imagery and its implications for understanding the motor system. Prog. Brain Res. 174, 219-229 (2009).

97. Clément, G. R., Bukley, A. P. \& Paloski, W. H. Artificial gravity as a countermeasure for mitigating physiological deconditioning during long-duration space missions. Front. Syst. Neurosci. 9, 92, http://www.ncbi.nlm.nih.gov/pubmed/ 26136665 \nhttp://www.pubmedcentral.nih.gov/articlerender.fcgi?artid=PMC44 70275 (2015).

98. Homick J. L., Delaney P., Rodda K. Overview of the Neurolab Spacelab mission Acta Astronaut. 42, 69-87 (1998).

99. Mechelli, A., Price, C. J., Friston, K. J. \& Ashburner, J. Voxel-based morphometry of the human brain: methods and applications. Curr. Med. Imaging Rev. 1, 105-113 (2005).

100. Popova, N. et al. Risk neurogenes for long-term spaceflight: dopamine and serotonin brain system. Mol. Neurobiol. 51, 1443-1451 (2015).

101. Marrosu, F. et al. Microdialysis measurement of cortical and hippocampal acetylcholine-release during sleep-wake cycle in freely moving cats. Brain. Res. [Internet] 671, 329-332 (1995). 
102. Baxter, M. G. \& Chiba, A. A. Cognitive functions of the basal forebrain. Curr. Opin. Neurobiol. 9, 178-183, http://www.sciencedirect.com/science/article/pii/ S0959438899800245 (1999).

103. Giovannini, M. et al. Effects of novelty and habituation on acetylcholine, GABA, and glutamate release from the frontal cortex and hippocampus of freely moving rats. Neuroscience. 106, 43-53 (2001).

104. Fibiger, H. C., Damsma, G. \& Day, J. C. Behavioral pharmacology and biochemistry of central cholinergic neurotransmission. Adv. Exp. Med. Biol. [Internet] 295, 399-414, http://www.ncbi.nlm.nih.gov/entrez/query.fcgi?cmd=Retrieve\&db= PubMed\&dopt $=$ Citation\&list_uids $=1663698$ (1991).

105. Taubert, M. et al. Dynamic properties of human brain structure: learning-related changes in cortical areas and associated fiber connections. J. Neurosci. 30, 11670-11677, http://www.jneurosci.org/content/30/35/11670\&n http://www.jneurosci.org/content/30/35/11670.full\&nhttp://www.jneurosci.org/ content/30/35/11670.full.pdf\&nhttp://www.ncbi.nlm.nih.gov/pubmed/20810 887 (2010).

106. Good, C. D. et al. A voxel-based morphometric study of ageing in 465 normal adult human brains. Neuroimage 14, 21-36 (2001).

107. Salthouse, T. The processing-speed theory of adult age differences in cognition. Psychol. Rev. 103, 403-428 (1996).
108. Salthouse, T. What and when of cognitive aging. Curr. Dir. Psychol. Sci. 13, 140-144 (2004).

109. Uludağ K, Roebroeck A. General overview on the merits of multimodal neuroimaging data fusion. Neuroimage, 1-8, http://www.ncbi.nlm.nih.gov/pubmed/ 24845622 (2014)

110. Basner, M. et al. Development and validation of the cognition test battery for spaceflight. Aerosp. Med. Hum. Perform. 86, 942-952 (2015).

111. Duvernoy H. The Human Brain: Surface, Blood Supply, and Three-Dimensional Sectional Anatomy. 2nd edn (Springer-Verlag, 1999).

cc) (i)

This work is licensed under a Creative Commons Attribution 4.0 International License. The images or other third party material in this article are included in the article's Creative Commons license, unless indicated otherwise in the credit line; if the material is not included under the Creative Commons license, users will need to obtain permission from the license holder to reproduce the material. To view a copy of this license, visit http://creativecommons.org/licenses/by/4.0/

(c) The Author(s) 2017 